\title{
Utility of adherence checks in patients with severe asthma eligible for biologics: a single centre retrospective analysis
}

Madeleine E. Oliver BA*, Sarah Poole BPharm MSc MRPharmS*, Catherine Borg RN MSc, Clare Connolly RN Msc, lan D Pavord DM FRCP FERS FMedSci, Timothy SC Hinks BMBCh MRCP, Simon Couillard MD FRCPC.

*MEO and SP contributed equally.

Introduction: United-Kingdom guidelines require good adherence to qualify for biologics, but reports suggest $25 \%$ of patients are nonadherent at time of initiation. The extent to which collaboration with a clinical pharmacist allows better fulfilment of guideline criteria has not been established.

Methods: We retrospectively analysed adherence checks done in the Oxford Special Airways Clinic (Oxford, UK) prior to initiation of biologic treatment for severe asthma between Dec 2013 and Aug 2020. Adherence to inhaled corticosteroid and maintenance oral corticosteroids (OCS) was defined as $\geq 75 \%$ of prescriptions collected out of the total expected in 1 year. Other guideline criteria for biologics are $\geq 3$ OCS bursts and/or $\geq 50 \%$ of previous year on maintenance OCS in optimally-treated severe asthma.

Results: 280 of 289 patients on biologics had a pre-biologic adherence check available. The median adherence to ICS was 100\% (IQR: $83 \%-100 \%$ ) and the median number of asthma attacks in the previous year was 3 (IQR 1-6). Overall adherence and compliance with pharmaceutical criteria for biologics was shown in 249 patients (89\%).

Conclusion: An adherence check by a clinical pharmacist prior to initiating a biologic for severe asthma is associated with $89 \%$ compliance to prescription guidelines, emphasising the importance of multidisciplinary work.

Characters with spaces

1409 